\title{
Hamiltonian Systems Inspired by the Schrödinger Equation ${ }^{\star}$
}

Vasyl KOVALCHUK and Jan Jerzy SEAWIANOWSKI

Institute of Fundamental Technological Research, Polish Academy of Sciences, 21, Świȩtokrzyska str., 00-049 Warsaw, Poland

E-mail:vkoval@ippt.gov.pl,jslawian@ippt.gov.pl

URL: http://www.ippt.gov.pl/ vkoval/, http://www.ippt.gov.pl/ jslawian/

Received October 30, 2007, in final form April 25, 2008; Published online May 27, 2008

Original article is available at http://www.emis.de/journals/SIGMA/2008/046/

\begin{abstract}
Described is $n$-level quantum system realized in the $n$-dimensional "Hilbert" space $H$ with the scalar product $G$ taken as a dynamical variable. The most general Lagrangian for the wave function and $G$ is considered. Equations of motion and conservation laws are obtained. Special cases for the free evolution of the wave function with fixed $G$ and the pure dynamics of $G$ are calculated. The usual, first- and second-order modified Schrödinger equations are obtained.
\end{abstract}

Key words: Schrödinger equation; Hamiltonian systems on manifolds of scalar products; $n$-level quantum systems; scalar product as a dynamical variable; essential non-perturbative nonlinearity; conservation laws; GL $(n, \mathbb{C})$-invariance

2000 Mathematics Subject Classification: 81P05; 81R05; 81Q99; 37J05; 15A04; 15A63; 15A90; 20G20

\section{Introduction}

Configuration spaces endowed with some algebraic structures are of interest in various areas of mathematical physics. As a rule, Hamiltonian systems defined on their cotangent bundles have certain mathematically and physically interesting features, especially when their Hamiltonians are somehow suited to the mentioned algebraic structures, e.g., are invariant under their automorphism groups or subgroups. The best known example is the theory of Hamiltonian systems on the cotangent bundles of Lie groups or their group spaces (or even more general homogeneous spaces) where by the group space we mean the homogeneous space with trivial isotropy groups, i.e., groups which "forgot" about having the distinguished neutral element. The special attention in applications is paid to Hamiltonians invariant under left or right translations or under both of them. The examples are the rigid bodies, incompressible ideal fluids [1], affinely-rigid bodies (see for example $[13,14]$ and references therein), etc.

Usually in physics one deals with linear groups, i.e., groups faithfully realizable by finite matrices. The only relatively known exceptions are $\overline{\mathrm{GL}(n, \mathbb{R})}$ and $\overline{\mathrm{SL}(n, \mathbb{R})}$, i.e., the covering groups of $\mathrm{GL}(n, \mathbb{R})$ and $\mathrm{SL}(n, \mathbb{R})$ respectively. However, in spite of various attempts of $\mathrm{F}$. Hehl, Y. Ne'eman and others (see for example $[6,7]$ ), their physical applicability is as yet rather doubtful and questionable. So, one of the best known examples of Hamiltonian systems on algebraic structures are (usually invariant) ones on the cotangent bundles of matrix groups or more generally some matrix manifolds. From the purely algebraic point of view, such configuration spaces consist of second-order (and non-degenerate) tensors in some linear spaces. Geometrically they represent linear transformations. Some questions appear here in a natural way. Namely, it is

\footnotetext{
*This paper is a contribution to the Proceedings of the Seventh International Conference "Symmetry in Nonlinear Mathematical Physics" (June 24-30, 2007, Kyiv, Ukraine). The full collection is available at http://www.emis.de/journals/SIGMA/symmetry2007.html
} 
a rule that all second-order tensors, i.e., not only mixed ones, are of particular importance in physics. Twice covariant or contravariant tensors represent various scalar products, e.g., metric tensors, electromagnetic fields, gauge fields, etc. In a purely analytical sense all second-order tensors are matrices. Obviously, due to the difference in the transformation rules, all they are geometrically completely different objects. Nevertheless the natural question arises as to the existence of geometrically and physically interesting Hamiltonian systems on the cotangent bundles of manifolds of second-order tensors of other type than linear transformations. We mean here first of all the manifolds of scalar products, both real-symmetric and complex-sesquilinearhermitian. Also the twice covariant and contravariant tensors without any special symmetries may be interesting.

One of our motivations has to do with certain ideas concerning nonlinear quantum mechanics. Various ways towards nonlinearity in quantum case were presented, e.g., in the review papers $[15,16]$, from those motivated by paradoxes of the quantum measurement, the interplay of unitary evolution and reduction, etc., to certain ideas based on geometry like, for instance, the Doebner-Goldin nonlinearity $[2,3,5]$. However in this article we are motivated by another idea. Namely, it is well known that the unitary evolution of a quantum system, described by the Schrödinger equation, may be interpreted as a Hamiltonian system on Hilbert space. The most convenient way to visualize this is to start from finite-dimensional, i.e., " $n$-level", quantum systems $(n<\infty)$. The scalar product is then fixed once for all and is an absolute element of the system. The true "degrees of freedom" are represented only by the vector of the underlying Hilbert space "wave functions". And here some natural analogy appears with the situation in Special Relativity vs. General Relativity:

- In specially-relativistic theories the metric tensor is fixed once for all as an absolute object, whereas all physical fields are "flexible" and satisfy differential equations as a rule derivable from the variational principle. The fixed metric tensor is then used as a "glue" to contract tensor indices in order to build the scalar density of weight one dependent algebraically on fields and their first-order derivatives.

- In generally-relativistic theories the metric tensor becomes flexible as well, it is included to degrees of freedom and satisfies differential equations together with the other "physical" fields. Moreover it becomes itself the physical field, in this case the gravitational one.

One can wonder whether one should not follow a similar pattern in quantum mechanics. Just to make the scalar product "flexible" and dynamically coupled to the $\psi$-object, i.e., to the "wave function". But, as mentioned, the scalar product is a twice covariant tensor. And so we return to the idea of Hamiltonian systems on manifolds of scalar products or more general twice covariant or twice contravariant tensors. And the point is that such manifolds carry some natural Riemannian, pseudo-Riemannian or hermitian metric structures (almost canonical) which are essentially non-Euclidean, i.e., describe some curved geometries on manifolds of scalar products. Because of this the coefficients at their derivatives in Lagrangians (as quadratic forms of those velocities) are irreducibly non-constant. The resulting Euler-Lagrange equations for them, and therefore also for the systems "wave function + flexible scalar products", are essentially nonlinear. This is the non-perturbative nonlinearity, i.e., it cannot be interpreted as an artificial extra correction to some basic linear background. So, physically this is one of natural candidates for the effective and geometrically interpretable nonlinearity in quantum mechanics, perhaps somehow explaining the conflict between unitary evolution and reduction, which exists essentially due to the linearity of the standard quantum mechanics.

Beside the above-mentioned physical motivations, one should also stress that such Hamiltonian models are interesting in themselves from the purely geometric point of view. They are somehow similar to the (pseudo-)Riemannian metric structures on semisimple Lie groups, in particular to the Killing tensor. Nevertheless their algebraic and geometric structure is different. 
As to our knowledge, such Riemannian geometries have not been yet studied in mathematics. One has the feeling that being so canonical as Killing metrics on groups they may have some interesting geometric properties and are worth to be investigated.

\section{General problem}

Let us take a set of $n$ elements and some function $\psi$ defined on it, i.e.,

$$
\mathcal{N}=\{1, \ldots, n\} \in \mathbb{N}, \quad \psi: \mathcal{N} \rightarrow \mathbb{C} .
$$

Then we can define the "wave function" of the $n$-level quantum system as a following $n$-vector

$$
\psi=\left[\begin{array}{c}
\psi^{1} \\
\vdots \\
\psi^{n}
\end{array}\right], \quad \psi^{a}=\psi(a) \in \mathbb{C} .
$$

Let $H$ be a unitary space with the scalar product

$$
G: H \times H \rightarrow \mathbb{C},
$$

which is a sesquilinear hermitian form. Then such an $H$ will be our $n$-dimensional "Hilbert" space $\left(\mathbb{C}^{n}\right)$.

So, let us consider the general Lagrangian

$$
\begin{aligned}
L= & \alpha_{1} i G_{\bar{a} b}\left(\overline{\psi^{\bar{a}}} \dot{\psi}^{b}-\dot{\bar{\psi}}^{\bar{a}} \psi^{b}\right)+\alpha_{2} G_{\bar{a} b} \dot{\overline{\psi^{a}}} \dot{\psi}^{b}+\left[\alpha_{4} G_{\bar{a} b}+\alpha_{5} H_{\bar{a} b}\right] \bar{\psi}^{\bar{a}} \psi^{b} \\
& +\alpha_{3}\left[G^{b \bar{a}}+\alpha_{9} \bar{\psi}^{\bar{a}} \psi^{b}\right] \dot{G}_{\bar{a} b}+\Omega[\psi, G]^{d \bar{c} b \bar{a}} \dot{G}_{\bar{a} b} \dot{G}_{\bar{c} d}-\mathcal{V}(\psi, G),
\end{aligned}
$$

where

$$
\begin{aligned}
\Omega[\psi, G]^{d \bar{c} b \bar{a}}= & \alpha_{6}\left[G^{d \bar{a}}+\alpha_{9} \bar{\psi}^{\bar{a}} \psi^{d}\right]\left[G^{b \bar{c}}+\alpha_{9} \bar{\psi}^{\bar{c}} \psi^{b}\right]+\alpha_{7}\left[G^{b \bar{a}}+\alpha_{9} \bar{\psi}^{\bar{a}} \psi^{b}\right]\left[G^{d \bar{c}}+\alpha_{9} \bar{\psi}^{\bar{c}} \psi^{d}\right] \\
& +\alpha_{8} \bar{\psi}^{\bar{a}} \psi^{b} \bar{\psi}^{\bar{c}} \psi^{d}, \quad \Omega[\psi, G]^{d \bar{c} b \bar{a}}=\Omega[\psi, G]^{b \bar{a} d \bar{c}}
\end{aligned}
$$

and the potential $\mathcal{V}$ can be taken, for instance, in the following quartic form

$$
\mathcal{V}(\psi, G)=\varkappa\left(G_{\bar{a} b} \bar{\psi}^{\bar{a}} \psi^{b}\right)^{2} .
$$

The first and second terms in (1) (those with $\alpha_{1}$ and $\alpha_{2}$ ) describe the free evolution of wave function $\psi$ while $G$ is fixed. The Lagrangian for trivial part of the linear dynamics (those with $\alpha_{4}$ ) can be also taken in the more general form $f\left(G_{\bar{a} b} \bar{\psi}^{\bar{a}} \psi^{b}\right)$, where $f: \mathbb{R} \rightarrow \mathbb{R}$. The term with $\alpha_{5}$ corresponds to the Schrödinger dynamics while $G$ is fixed and then

$$
H_{b}^{a}=G^{a \bar{c}} H_{\bar{c} b}
$$

is the usual Hamilton operator. If we properly choose the constants $\alpha_{1}$ and $\alpha_{5}$, then we obtain precisely the Schrödinger equation. The dynamics of the scalar product $G$ is described by the terms linear and quadratic in the time derivative of $G$. In the above formulae $\bar{\psi}^{\bar{a}}=\overline{\psi^{a}}$ denotes the usual complex conjugation and $\alpha_{i}, i=\overline{1,9}$, and $\varkappa$ are some constants.

Then applying the variational procedure we obtain the equations of motion as follows

$$
\begin{aligned}
\frac{\delta L}{\delta \bar{\psi}^{\bar{a}}}= & \alpha_{2} G_{\bar{a} b} \ddot{\psi}^{b}+\left(\alpha_{2} \dot{G}_{\bar{a} b}-2 \alpha_{1} i G_{\bar{a} b}\right) \dot{\psi}^{b}-2 \alpha_{8} \dot{G}_{\bar{a} b} \psi^{b} \dot{G}_{\bar{c} d} \bar{\psi}^{\bar{c}} \psi^{d} \\
& -2 \alpha_{9}\left(\alpha_{6} \dot{G}_{\bar{a} d} \dot{G}_{\bar{c} b}+\alpha_{7} \dot{G}_{\bar{a} b} \dot{G}_{\bar{c} d}\right) \psi^{b}\left(G^{d \bar{c}}+\alpha_{9} \bar{\psi}^{\bar{c}} \psi^{d}\right) \\
& +\left[\left(2 \varkappa G_{\bar{c} d} \bar{\psi}^{\bar{c}} \psi^{d}-\alpha_{4}\right) G_{\bar{a} b}-\alpha_{5} H_{\bar{a} b}-\left[\alpha_{3} \alpha_{9}+\alpha_{1} i\right] \dot{G}_{\bar{a} b}\right] \psi^{b}=0
\end{aligned}
$$


and

$$
\begin{aligned}
\frac{\delta L}{\delta G_{\bar{a} b}}= & 2 \Omega[\psi, G]^{b \bar{a} d \bar{c}} \ddot{G}_{\bar{c} d}+2 \dot{\Omega}[\psi, G]^{b \bar{a} d \bar{c}} \dot{G}_{\bar{c} d}+\left(2 \varkappa G_{\bar{c} d} \bar{\psi}^{\bar{c}} \psi^{d}-\alpha_{4}\right) \bar{\psi}^{\bar{a}} \psi^{b} \\
& +2 G^{d \bar{a}}\left[\alpha_{6} G^{b \bar{e}}\left(G^{f \bar{c}}+\alpha_{9} \bar{\psi}^{\bar{c}} \psi^{f}\right)+\alpha_{7} G^{b \bar{c}}\left(G^{f \bar{e}}+\alpha_{9} \bar{\psi}^{\bar{e}} \psi^{f}\right)\right] \dot{G}_{\bar{c} d} \dot{G}_{\bar{e} f} \\
& -\alpha_{2} \dot{\bar{\psi}}^{\bar{a}} \dot{\psi}^{b}+\left[\alpha_{3} \alpha_{9}+\alpha_{1} i\right] \dot{\bar{\psi}}^{\bar{a}} \psi^{b}+\left[\alpha_{3} \alpha_{9}-\alpha_{1} i\right] \bar{\psi}^{\bar{a}} \dot{\psi}^{b}=0
\end{aligned}
$$

where

$$
\begin{aligned}
\dot{\Omega}[\psi, G]^{b \bar{a} d \bar{c}}= & \alpha_{8}\left(\dot{\bar{\psi}}^{\bar{a}} \psi^{b} \bar{\psi}^{\bar{c}} \psi^{d}+\bar{\psi}^{\bar{a}} \dot{\psi}^{b} \bar{\psi}^{\bar{c}} \psi^{d}+\overline{\psi^{a}} \psi^{b} \dot{\bar{\psi}}^{\bar{c}} \psi^{d}+\bar{\psi}^{\bar{a}} \psi^{b} \bar{\psi}^{\bar{c}} \dot{\psi}^{d}\right) \\
& +\alpha_{6} \alpha_{9}\left(\left[\dot{\bar{\psi}}^{\bar{a}} \psi^{d}+\bar{\psi} \bar{\psi}^{\bar{a}} \dot{\psi}^{d}\right]\left[G^{b \bar{c}}+\alpha_{9} \bar{\psi}^{\bar{c}} \psi^{b}\right]+\left[\dot{\bar{\psi}}^{\bar{c}} \psi^{b}+\bar{\psi}^{\bar{c}} \dot{\psi}^{b}\right]\left[G^{d \bar{a}}+\alpha_{9} \bar{\psi}^{\bar{a}} \psi^{d}\right]\right) \\
& +\alpha_{7} \alpha_{9}\left(\left[\dot{\bar{\psi}}^{\bar{a}} \psi^{b}+\overline{\psi^{a}} \dot{\psi}^{b}\right]\left[G^{d \bar{c}}+\alpha_{9} \bar{\psi}^{\bar{c}} \psi^{d}\right]+\left[\dot{\bar{\psi}}^{\bar{c}} \psi^{d}+\bar{\psi}^{\bar{c}} \dot{\psi}^{d}\right]\left[G^{b \bar{a}}+\alpha_{9} \bar{\psi}^{\bar{a}} \psi^{b}\right]\right) \\
& -\alpha_{6}\left[G^{d \bar{e}} G^{f \bar{a}}\left(G^{b \bar{c}}+\alpha_{9} \bar{\psi}^{\bar{c}} \psi^{b}\right)+G^{b \bar{e}} G^{f \bar{c}}\left(G^{d \bar{a}}+\alpha_{9} \bar{\psi}^{\bar{a}} \psi^{d}\right)\right] \dot{G}_{\bar{e} f} \\
& -\alpha_{7}\left[G^{b \bar{e}} G^{f \bar{a}}\left(G^{d \bar{c}}+\alpha_{9} \bar{\psi}^{\bar{c}} \psi^{d}\right)+G^{d \bar{e}} G^{f \bar{c}}\left(G^{b \bar{a}}+\alpha_{9} \bar{\psi}^{\bar{a}} \psi^{b}\right)\right] \dot{G}_{\bar{e} f} .
\end{aligned}
$$

\section{Towards the canonical formalism}

The Legendre transformations leads us to the following canonical variables

$$
\begin{aligned}
& \pi_{b}=\frac{\partial L}{\partial \dot{\psi}^{b}}=\alpha_{2} G_{\bar{a} b} \dot{\bar{\psi}}^{\bar{a}}+\alpha_{1} i G_{\bar{a} b} \bar{\psi}^{\bar{a}}, \quad \bar{\pi}_{\bar{a}}=\frac{\partial L}{\partial \dot{\bar{\psi}}^{\bar{a}}}=\alpha_{2} G_{\bar{a} b} \dot{\psi}^{b}-\alpha_{1} i G_{\bar{a} b} \psi^{b}, \\
& \pi^{\bar{a} b}=\frac{\partial L}{\partial \dot{G}_{\bar{a} b}}=\alpha_{3}\left[G^{b \bar{a}}+\alpha_{9} \bar{\psi}^{\bar{a}} \psi^{b}\right]+2 \Omega[\psi, G]^{b \bar{a} d \bar{c}} \dot{G}_{\bar{c} d} .
\end{aligned}
$$

The energy of our $n$-level Hamiltonian system is as follows

$$
\begin{aligned}
E & =\dot{\bar{\psi}^{\bar{a}}} \frac{\partial L}{\partial \dot{\bar{\psi}}^{\bar{a}}}+\dot{\psi}^{b} \frac{\partial L}{\partial \dot{\psi}^{b}}+\dot{G}_{\bar{a} b} \frac{\partial L}{\partial \dot{G}_{\bar{a} b}}-L \\
& =\alpha_{2} G_{\bar{a} b} \dot{\bar{\psi}}^{\bar{a}} \dot{\psi}^{b}-\left(\alpha_{4} G_{\bar{a} b}+\alpha_{5} H_{\bar{a} b}\right) \bar{\psi}^{\bar{a}} \psi^{b}+\Omega[\psi, G]^{\bar{a} b \bar{c} d} \dot{G}_{\bar{a} b} \dot{G}_{\bar{c} d}+\varkappa\left(G_{\bar{a} b} \bar{\psi}^{\bar{a}} \psi^{b}\right)^{2} .
\end{aligned}
$$

Inverting the expressions (3), (4) we obtain that

$$
\begin{aligned}
& \dot{\bar{\psi}}^{\bar{a}}=\frac{1}{\alpha_{2}} G^{b \bar{a}} \pi_{b}-\frac{\alpha_{1}}{\alpha_{2}} i \bar{\psi}^{\bar{a}}, \quad \dot{\psi}^{b}=\frac{1}{\alpha_{2}} G^{b \bar{a}_{\bar{\pi}}} \bar{\pi}_{\bar{a}}+\frac{\alpha_{1}}{\alpha_{2}} i \psi^{b}, \\
& \dot{G}_{\bar{a} b}=\frac{1}{2} \Omega[\psi, G]_{\bar{a} b \bar{c} d}^{-1}\left(\pi^{\bar{c} d}-\alpha_{3}\left[G^{d \bar{c}}+\alpha_{9} \bar{\psi}^{\bar{c}} \psi^{d}\right]\right),
\end{aligned}
$$

where

$$
\begin{aligned}
& \Omega[\psi, G]_{\bar{a} b \bar{c} d}^{-1}=\Lambda[\psi, G]_{\bar{a} b \bar{c} d}^{-1}-\frac{\alpha_{8}}{1+\alpha_{8} \theta_{2}[\psi, G]} \Lambda[\psi, G]_{\bar{a} b \bar{e} f}^{-1} \bar{\psi}^{\bar{e}} \psi^{f} \Lambda[\psi, G]_{\bar{c} d \bar{g} h}^{-1} \bar{\psi}^{\bar{g}} \psi^{h}, \\
& \Lambda[\psi, G]_{\bar{a} b \bar{c} d}^{-1}=\frac{1}{\alpha_{6}} \lambda[\psi, G]_{\bar{a} d}^{-1} \lambda[\psi, G]_{\bar{c} b}^{-1}-\frac{\alpha_{7}}{\alpha_{6}\left(\alpha_{6}+n \alpha_{7}\right)} \lambda[\psi, G]_{\bar{a} b}^{-1} \lambda[\psi, G]_{\bar{c} d}^{-1}, \\
& \lambda[\psi, G]_{\bar{a} b}^{-1}=G_{\bar{a} b}-\frac{\alpha_{9}}{1+\alpha_{9} \theta_{1}[\psi, G]} G_{\bar{a} d} G_{\bar{c} b} \bar{\psi}^{\bar{c}} \psi^{d} \\
& \theta_{2}[\psi, G]=\Lambda[\psi, G]_{\bar{a} b \bar{c} d}^{-1} \bar{\psi}^{\bar{a}} \psi^{b} \bar{\psi}^{\bar{c}} \psi^{d}=\frac{\alpha_{6}+(n-1) \alpha_{7}}{\alpha_{6}\left(\alpha_{6}+n \alpha_{7}\right)}\left(\frac{\theta_{1}[\psi, G]}{1+\alpha_{9} \theta_{1}[\psi, G]}\right)^{2}, \\
& \theta_{1}[\psi, G]=G_{\bar{a} b} \bar{\psi}^{\bar{a}} \psi^{b}
\end{aligned}
$$


and then the Hamiltonian has the following form

$$
\begin{aligned}
H= & \frac{1}{\alpha_{2}} G^{b \bar{a}} \bar{\pi}_{\bar{a}} \pi_{b}+\frac{\alpha_{1}}{\alpha_{2}} i\left(\psi^{b} \pi_{\psi b}-\bar{\psi}^{\bar{a}} \bar{\pi}_{\bar{a}}\right)-\left[\left(\alpha_{4}-\frac{\alpha_{1}^{2}}{\alpha_{2}}\right) G_{\bar{a} b}+\alpha_{5} H_{\bar{a} b}\right] \bar{\psi}^{\bar{a}} \psi^{b} \\
& +\frac{1}{4} \Omega[\psi, G]_{\bar{a} b \bar{c} d}^{-1} \pi^{\bar{a} b} \pi^{\bar{c} d}-\frac{\alpha_{3}}{2} \Omega[\psi, G]_{\bar{a} b \bar{c} d}^{-1}\left[G^{b \bar{a}}+\alpha_{9} \overline{\psi^{\bar{a}}} \psi^{b}\right] \pi^{\bar{c} d} \\
& +\frac{\alpha_{3}^{2}}{4} \Omega[\psi, G]_{\bar{a} b \bar{c} d}^{-1}\left[G^{b \bar{a}}+\alpha_{9} \bar{\psi}^{\bar{a}} \psi^{b}\right]\left[G^{d \bar{c}}+\alpha_{9} \bar{\psi}^{\bar{c}} \psi^{d}\right]+\varkappa\left(G_{\bar{a} b} \bar{\psi}^{\bar{a}} \psi^{b}\right)^{2}
\end{aligned}
$$

\section{Special cases}

\subsection{Pure dynamics for $G$}

First of all, if we consider the pure dynamics of scalar product $G$ while the wave function $\psi$ is fixed, then from (2) we obtain the following equations of motion

$$
\begin{aligned}
\Omega[\psi, G]^{b \bar{a} d \bar{c}} \ddot{G}_{\bar{c} d}= & \left(\frac{\alpha_{4}}{2}-\varkappa \theta_{1}[\psi, G]\right) \bar{\psi}^{\bar{a}} \psi^{b}+\alpha_{7} \theta_{3}[\psi, G]\left(G^{b \bar{a}}+\alpha_{9} \bar{\psi}^{\bar{a}} \psi^{b}\right) \\
& +\alpha_{6} \dot{G}_{\bar{c} d} \dot{G}_{\bar{e} f}\left(\gamma[\psi, G]^{b \bar{e} f \bar{c} d \bar{a}}+\gamma[\psi, G]^{f \bar{a} d \bar{e} b \bar{c}}-\gamma[\psi, G]^{b \bar{e} d \bar{a} f \bar{c}}\right),
\end{aligned}
$$

where

$$
\theta_{3}[\psi, G]=G^{d \bar{e}} G^{f \bar{c}} \dot{G}_{\bar{c} d} \dot{G}_{\bar{e} f}, \quad \gamma[\psi, G]^{f \bar{e} d \bar{c} b \bar{a}}=G^{f \bar{e}} G^{d \bar{c}}\left(G^{b \bar{a}}+\alpha_{9} \bar{\psi}^{\bar{a}} \psi^{b}\right) .
$$

If we additionally suppose that $\alpha_{4}=\alpha_{8}=\alpha_{9}=\varkappa=0$, then (5) simplifies significantly

$$
\left(\alpha_{6} G^{b \bar{c}} G^{d \bar{a}}+\alpha_{7} G^{b \bar{a}} G^{d \bar{c}}\right)\left(\ddot{G}_{\bar{c} d}-\dot{G}_{\bar{c} f} G^{f \bar{e}} \dot{G}_{\bar{e} d}\right)=0 .
$$

Hence, the pure dynamics of the scalar product is described by the following equations

$$
\ddot{G}_{\bar{a} b}-\dot{G}_{\bar{a} d} G^{d \bar{c}} \dot{G}_{\bar{c} b}=0 .
$$

Let us now demand that $\dot{G} G^{-1}$ is equal to some constant value $E$, i.e., $\dot{G}=E G$, then

$$
\ddot{G}=E \dot{G}=E^{2} G
$$

and

$$
\dot{G} G^{-1} \dot{G}=E G G^{-1} E G=E^{2} G
$$

therefore our equations of motion (6) are fulfilled automatically and the solution is as follows

$$
G(t)_{\bar{a} b}=(\exp (E t))^{\bar{c}}{ }_{\bar{a}} G_{0 \bar{c} b} .
$$

Similarly if we demand that $G^{-1} \dot{G}$ is equal to some other constant $E^{\prime}$, i.e., $\dot{G}=G E^{\prime}$,

$$
\begin{aligned}
& \ddot{G}=\dot{G} E^{\prime 2}=G E^{\prime 2}, \\
& \dot{G} G^{-1} \dot{G}=G E^{\prime} G^{-1} G E^{\prime}=G E^{\prime 2},
\end{aligned}
$$

then the equations of motion are also fulfilled and the solution is as follows

$$
G(t)_{\bar{a} b}=G_{0 \bar{a} d}\left(\exp \left(E^{\prime} t\right)\right)_{b}^{d}
$$

The connection between these two different constants $E$ and $E^{\prime}$ is written below

$$
\dot{G}(0)=\dot{G}_{0}=G_{0} E^{\prime}=E G_{0} .
$$




\subsection{Usual and first-order modified Schrödinger equations}

The second interesting special case is obtained when we suppose that the scalar product $G$ is fixed, i.e., the equations of motion are as follows

$$
\alpha_{2} \ddot{\psi}^{a}-2 \alpha_{1} i \dot{\psi}^{a}+\left(2 \varkappa \theta_{1}[\psi, G]-\alpha_{4}\right) \psi^{a}-\alpha_{5} H_{b}^{a} \psi^{b}=0 \text {. }
$$

Then if we also take all constants of model to be equal to 0 except of the following ones

$$
\alpha_{1}=\frac{\hbar}{2}, \quad \alpha_{5}=-1
$$

we end up with the well-known usual Schrödinger equation

$$
i \hbar \dot{\psi}^{a}=H_{b}^{a} \psi^{b} .
$$

Its first-order modified version is obtained when we suppose that $G$ is a dynamical variable and $\alpha_{2}$ is equal to 0 , i.e.,

$$
\begin{aligned}
& i \hbar \dot{\psi}^{a}=H_{b}^{a} \psi^{b}-\left[\frac{i \hbar}{2}+\alpha_{3} \alpha_{9}\right] G^{a \bar{c}} \dot{G}_{\bar{c} b} \psi^{b}+\left(2 \varkappa \theta_{1}[\psi, G]-\alpha_{4}\right) \psi^{a} \\
& -2 \alpha_{8} G^{a \bar{c}} \dot{G}_{\bar{c} b} \psi^{b} \dot{G}_{\bar{e} d} \bar{\psi}^{\bar{e}} \psi^{d}-2 \alpha_{9} G^{a \bar{c}}\left(\alpha_{6} \dot{G}_{\bar{c} d} \dot{G}_{\bar{e} b}+\alpha_{7} \dot{G}_{\bar{c} b} \dot{G}_{\bar{e} d}\right) \psi^{b}\left(G^{d \bar{e}}+\alpha_{9} \bar{\psi}^{\bar{e}} \psi^{d}\right) \text {, } \\
& 2 \Omega[\psi, G]^{b \bar{a} d \bar{c}} \ddot{G}_{\bar{c} d}=\left[\frac{i \hbar}{2}-\alpha_{3} \alpha_{9}\right] \bar{\psi}^{\bar{a}} \dot{\psi}^{b}-\left[\frac{i \hbar}{2}+\alpha_{3} \alpha_{9}\right] \dot{\bar{\psi}}^{\bar{a}} \psi^{b} \\
& -2 G^{d \bar{a}}\left[\alpha_{6} G^{b \bar{e}}\left(G^{f \bar{c}}+\alpha_{9} \bar{\psi}^{\bar{c}} \psi^{f}\right)+\alpha_{7} G^{b \bar{c}}\left(G^{f \bar{e}}+\alpha_{9} \bar{\psi}^{\bar{e}} \psi^{f}\right)\right] \dot{G}_{\bar{c} d} \dot{G}_{\bar{e} f} \\
& -\left(2 \varkappa \theta_{1}[\psi, G]-\alpha_{4}\right) \bar{\psi}^{\bar{a}} \psi^{b}-2 \dot{\Omega}[\psi, G]^{b \bar{a} d \bar{c}} \dot{G}_{\bar{c} d} \text {. }
\end{aligned}
$$

We can rewrite (8) in the following form

$$
i \hbar \dot{\psi}^{a}=H_{\text {eff }}^{a} b \psi^{b}
$$

where the effective Hamilton operator is given as follows:

$$
\begin{aligned}
H_{\mathrm{eff}}{ }^{a}{ }_{b}= & H^{a}{ }_{b}-\left[\frac{i \hbar}{2}+\alpha_{3} \alpha_{9}\right] G^{a \bar{c}} \dot{G}_{\bar{c} b}+\left(2 \varkappa \theta_{1}[\psi, G]-\alpha_{4}\right) \delta^{a}{ }_{b}-2 \alpha_{8} G^{a \bar{c}} \dot{G}_{\bar{c} b} \dot{G}_{\bar{e} d} \bar{\psi}^{\bar{e}} \psi^{d} \\
& -2 \alpha_{9} G^{a \bar{c}}\left(\alpha_{6} \dot{G}_{\bar{c} d} \dot{G}_{\bar{e} b}+\alpha_{7} \dot{G}_{\bar{c} b} \dot{G}_{\bar{e} d}\right)\left(G^{d \bar{e}}+\alpha_{9} \bar{\psi}^{\bar{e}} \psi^{d}\right) .
\end{aligned}
$$

\subsection{Second-order modified Schrödinger equation}

The idea of introducing the second time derivative of the wave function into the usual Schrödinger equation as a correction term is not completely new and has been already discussed in the literature. The similar problems were studied a long time ago by A. Barut and more recently have been re-investigated by V.V. Dvoeglazov, S. Kruglov, J.P. Vigier and others (see, e.g., [4, 10] and references therein; the authors of this article are grateful to one of the referee for pointing them to above-mentioned references). Among others there is also an interesting article where the authors used the analogy between the Schrödinger and Fourier equations [11].

The quantum Fourier equation which describes the heat (mass) diffusion on the atomic level has the following form

$$
\frac{\partial T}{\partial t}=\frac{\hbar}{m} \nabla^{2} T
$$


If we make the substitutions $t \rightarrow i t / 2$ and $T \rightarrow \psi$, then we end up with the free Schrödinger equation

$$
i \hbar \frac{\partial \psi}{\partial t}=-\frac{\hbar^{2}}{2 m} \nabla^{2} \psi
$$

The complete Schrödinger equation with the potential term $V$ after the reverse substitutions $t \rightarrow-2 i t$ and $\psi \rightarrow T$ gives us the parabolic quantum Fokker-Planck equation, which describes the quantum heat transport for $\Delta t>\tau$, where $\tau=\hbar / m \alpha^{2} c^{2} \sim 10^{-17}$ sec and $c \tau \sim 1 \mathrm{~nm}$, i.e.,

$$
\frac{\partial T}{\partial t}=\frac{\hbar}{m} \nabla^{2} T-\frac{2 V}{\hbar} T .
$$

For ultrashort time processes when $\Delta t<\tau$ one obtains the generalized quantum hyperbolic heat transport equation

$$
\tau \frac{\partial^{2} T}{\partial t^{2}}+\frac{\partial T}{\partial t}=\frac{\hbar}{m} \nabla^{2} T-\frac{2 V}{\hbar} T
$$

(its structure and solutions for ultrashort thermal processes were investigated in [9]) which leads us to the second-order modified Schrödinger equation

$$
2 \tau \hbar \frac{\partial^{2} \psi}{\partial t^{2}}+i \hbar \frac{\partial \psi}{\partial t}=-\frac{\hbar^{2}}{2 m} \nabla^{2} \psi+V \psi
$$

in which the additional term describes the interaction of electrons with surrounding space-time

filled with virtual positron-electron pairs. It is easy to see that (9) is analogous to (7) if we suppose that

$$
\alpha_{1}=\frac{\hbar}{2}, \quad \alpha_{2}=-2 \tau \hbar, \quad \alpha_{4}=0, \quad \alpha_{5}=-1, \quad \varkappa=0 .
$$

\section{Conservation laws and $\operatorname{GL}(n, \mathbb{C})$-invariance}

So, if we investigate the invariance of our general Lagrangian (1) under the group $\operatorname{GL}(n, \mathbb{C})$ and consider some one-parameter group of transformations

$$
\{\exp (A \tau): \tau \in \mathbb{R}\}, \quad A \in \mathrm{L}(n, \mathbb{C}),
$$

then the infinitesimal transformations rules for $\psi$ and $G$ are as follows

$$
\psi^{a} \mapsto L_{b}^{a} \psi^{b}, \quad G^{a \bar{c}} \mapsto L_{b}^{a} \bar{L}_{\bar{e}}^{\bar{c}} G^{b \bar{e}}, \quad G_{\bar{a} b} \mapsto G_{\bar{c} d}{\overline{L^{-1}}}_{\bar{a}}^{\bar{c}} L^{-1 d_{b}},
$$

where

$$
L^{a}{ }_{b}=\delta^{a}{ }_{b}+\epsilon A_{b}^{a}, \quad L^{-1 a}{ }_{b} \approx \delta^{a}{ }_{b}-\epsilon A_{b}^{a}, \quad \epsilon \approx 0 .
$$

So leaving only the first-order terms with respect to $\epsilon$ we obtain that the variations of $\psi$ and $G$ are as follows

$$
\begin{aligned}
& \delta \psi^{a}=\epsilon A_{b}^{a} \psi^{b}, \quad \delta \bar{\psi}^{\bar{a}}=\epsilon \bar{A}^{\bar{a}} \bar{c}_{\bar{c}} \bar{\psi}^{\bar{c}}, \\
& \delta G^{a \bar{c}}=\epsilon\left(A^{a}{ }_{b} G^{b \bar{c}}+\bar{A}^{\bar{c}}{ }_{\bar{e}}^{a} G^{a \bar{e}}\right), \quad \delta G_{\bar{a} b}=-\epsilon\left(G_{\bar{c} b} \bar{A}^{\bar{c}}{ }_{\bar{a}}+G_{\bar{a} d} A_{b}^{d}\right),
\end{aligned}
$$

then

$$
\frac{1}{\epsilon}\left(\frac{\partial L}{\partial \dot{\bar{\psi}}^{\bar{a}}} \delta \bar{\psi}^{\bar{a}}+\frac{\partial L}{\partial \dot{\psi}^{b}} \delta \psi^{b}\right)=G_{\bar{a} b}\left(\alpha_{2} \dot{\bar{\psi}}^{\bar{a}}+\alpha_{1} i \bar{\psi}^{\bar{a}}\right) A^{b}{ }_{d} \psi^{d}+G_{\bar{a} b}\left(\alpha_{2} \dot{\psi}^{b}-\alpha_{1} i \psi^{b}\right) \bar{A}^{\bar{a}} \overline{\bar{c}}^{\bar{c}}
$$


and

$$
\begin{aligned}
\frac{1}{\epsilon} \frac{\partial L}{\partial \dot{G}_{\bar{a} b}} \delta G_{\bar{a} b}= & -\left[\alpha_{3}\left(\delta^{b}{ }_{f}+\alpha_{9} G_{\bar{a} f} \bar{\psi}^{\bar{a}} \psi^{b}\right)+2 \Omega[\psi, G]^{b \bar{a} d \bar{c}} G_{\bar{a} f} \dot{G}_{\bar{c} d}\right] A^{f}{ }_{b} \\
& -\left[\alpha_{3}\left(\delta^{\bar{a}}{ }_{\bar{e}}+\alpha_{9} G_{\bar{e} b} \bar{\psi}^{\bar{a}} \psi^{b}\right)+2 \Omega[\psi, G]^{b \bar{a} d \bar{c}} G_{\bar{e} b} \dot{G}_{\bar{c} d}\right] \bar{A}^{\bar{e}}{ }_{\bar{a}}
\end{aligned}
$$

If we consider some fixed scalar product $G_{0}$ and take the $G_{0}$-hermitian $A$ 's, then

$$
A_{b}^{a}=G_{0}^{a \bar{c}} \widetilde{A}_{\bar{c} b}, \quad \bar{A}_{\bar{c}}^{\bar{a}}=\widetilde{A}_{\bar{c} b} G_{0}^{b \bar{a}}, \quad \widetilde{A}^{\dagger}=\widetilde{A},
$$

and therefore the expressions (10) and (11) are written together in the matrix form as follows

$$
\mathcal{J}(A)=\operatorname{Tr}(V \widetilde{A})
$$

where the hermitian tensor $V$ describing the system of conserved physical quantities is given as follows

$$
\begin{aligned}
V= & \alpha_{2}\left(\psi \dot{\psi}^{\dagger} G G_{0}^{-1}+G_{0}^{-1} G \dot{\psi} \psi^{\dagger}\right)+\left(\alpha_{1} i-\alpha_{3} \alpha_{9}\right) \psi \psi^{\dagger} G G_{0}^{-1} \\
& -\left(\alpha_{1} i+\alpha_{3} \alpha_{9}\right) G_{0}^{-1} G \psi \psi^{\dagger}-2 \alpha_{3} G_{0}^{-1}-2\left(G_{0}^{-1} G \omega[\psi, G]+\omega[\psi, G] G G_{0}^{-1}\right),
\end{aligned}
$$

where

$$
\omega[\psi, G]^{b \bar{a}}=\Omega[\psi, G]^{b \bar{a} d \bar{c}} \dot{G}_{\bar{c} d}
$$

Similarly for the $G_{0}$-antihermitian $A$ 's, i.e., when $\widetilde{A}^{\dagger}=-\widetilde{A}$, we obtain another hermitian tensor $W$ as a conserved value

$$
\mathcal{J}(A)=\operatorname{Tr}(i W \widetilde{A})
$$

where

$$
\begin{aligned}
i W= & \alpha_{2}\left(\psi \dot{\psi}^{\dagger} G G_{0}^{-1}-G_{0}^{-1} G \dot{\psi} \psi^{\dagger}\right)+\left(\alpha_{1} i-\alpha_{3} \alpha_{9}\right) \psi \psi^{\dagger} G G_{0}^{-1} \\
& +\left(\alpha_{1} i+\alpha_{3} \alpha_{9}\right) G_{0}^{-1} G \psi \psi^{\dagger}+2\left(G_{0}^{-1} G \omega[\psi, G]-\omega[\psi, G] G G_{0}^{-1}\right) .
\end{aligned}
$$

\section{Final remarks}

This is a very preliminary, simplified finite-level model. It is still not clear whether it is consistent with the usual statistical interpretation of quantum mechanics. This model is thought on as a step towards discussing the wave equations obtained by combining the first and second time derivatives. There are some indications that such a combination might be reasonable. Within a rather different context (motivated by the idea of conformal invariance) we studied such a problem in $[8,12]$ where the wave equations with the superposition of Dirac and d'Alembert operators were considered.

\section{Acknowledgements}

This paper contains results obtained within the framework of the research project 501018 32/1992 financed from the Scientific Research Support Fund in 2007-2010. The authors are greatly indebted to the Ministry of Science and Higher Education for this financial support.

The authors are also very grateful to the referees for their valuable remarks and comments concerning this article. 


\section{References}

[1] Arnold V.I., Mathematical methods of classical mechanics, Springer Graduate Texts in Mathematics, Vol. 60, Springer-Verlag, New York, 1978.

[2] Doebner H.-D., Goldin G.A., Introducing nonlinear gauge transformations in a family of nonlinear Schrödinger equations, Phys. Rev. A 54 (1996), 3764-3771.

[3] Doebner H.-D., Goldin G.A., Nattermann P., Gauge transformations in quantum mechanics and the unification of nonlinear Schrödinger equations, J. Math. Phys. 40 (1999), 49-63, quant-ph/9709036.

[4] Dvoeglazov V.V., The Barut second-order equation, dynamical invariants and interactions, J. Phys. Conf. Ser. 24 (2005), 236-240, math-ph/0503008.

[5] Goldin G.A., Gauge transformations for a family of nonlinear Schrödinger equations, J. Nonlinear Math. Phys. 4 (1997), 6-11.

[6] Hehl F.W., Kerlick G.D., von der Heyde P., General relativity with spin and torsion and its deviations from Einstein's theory, Phys. Rev. D 10 (1974), 1066-1069.

[7] Hehl F.W., Lord E.A., Ne'eman Y., Hadron dilatation, shear and spin as components of the intrinsic hypermomentum current and metric-affine theory of gravitation, Phys. Lett. B $\mathbf{7 1}$ (1977), 432-434.

[8] Kovalchuk V., Green function for Klein-Gordon-Dirac equation, J. Nonlinear Math. Phys. 11 (2004), suppl., $72-77$.

[9] Kozlowski M., Marciak-Kozlowska J., From quarks to bulk matter, Hadronic Press, USA, 2001.

[10] Kruglov S.I., On the generalized Dirac equation for fermions with two mass states, Ann. Fond. Louis de Broglie 29 (2004), 1005-1016, quant-ph/0408056.

[11] Marciak-Kozlowska J., Kozlowski M., Schrödinger equation for nanoscience, cond-mat/0306699.

[12] Sławianowski J.J., Kovalchuk V., Klein-Gordon-Dirac equation: physical justification and quantization attempts, Rep. Math. Phys. 49 (2002), 249-257.

[13] Sławianowski J.J., Kovalchuk V., Sławianowska A., Gołubowska B., Martens A., Rożko E.E., Zawistowski Z.J., Affine symmetry in mechanics of collective and internal modes. Part I. Classical models, Rep. Math. Phys. 54 (2004), 373-427, arXiv:0802.3027.

[14] Sławianowski J.J., Kovalchuk V., Sławianowska A., Gołubowska B., Martens A., Rożko E.E., Zawistowski Z.J., Affine symmetry in mechanics of collective and internal modes. Part II. Quantum models, Rep. Math. Phys. 55 (2005), 1-45, arXiv:0802.3028.

[15] Svetlichny G., Informal resource letter - nonlinear quantum mechanics on arXiv up to August 2004, quant-ph/0410036.

[16] Svetlichny G., Nonlinear quantum mechanics at the Planck scale, Internat. J. Theoret. Phys. 44 (2005), 2051-2058, quant-ph/0410230. 